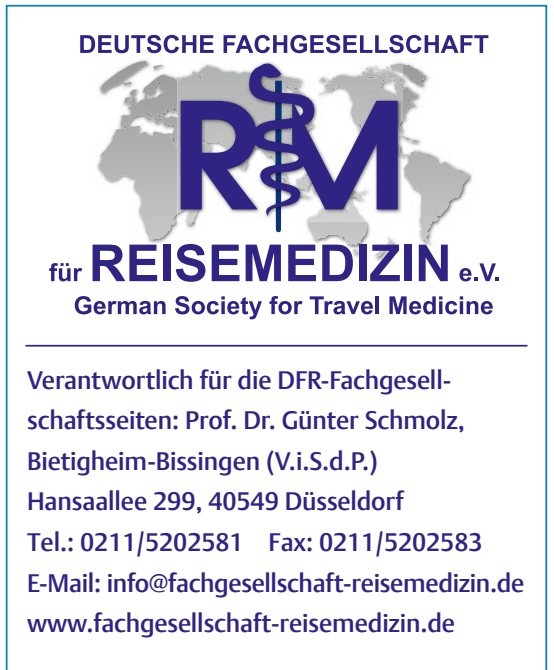

DEUTSCHE FACHGESELLSCHAFT

REISEMEDIZIN

Verantwortlich für die DFR-Fachgesell-

schaftsseiten: Prof. Dr. Günter Schmolz.

Tel.: 0211/5202581 Fax: 0211/5202583

www.fachgesellschaft-reisemedizin.de

\title{
Liebe Mitglieder der DFR, sehr verehrte Frau Kollegin, sehr geehrter Herr Kollege,
}

mikrobiologische und Schwermetallbelastung im Trinkwasser des South Luangwa Valley, Sambia, humanpathogene Rickettsien in Zecken des touristisch erschlossenen Ostens, ebenfalls Sambia, medizinische Diagnosen deutscher Geschäftsreisender nach China - Risiken neben der Impfsprechstunde: Das waren die Themen der Dissertationen, die am 24. September, zum Abschluss unserer Jahrestagung in Ludwigshafen, mit dem Erich-Kroeger-und-Klaus-Jörg-VolkmerPreis für Reisemedizin ausgezeichnet wurden. Die Arbeiten aus Sambia, eingereicht von Celina Drewell und Simone Kattner, entstammen der Abteilung von Prof. Thomas Küpper, Universität Aachen, die Arbeit zu Gesundheitsrisiken von Geschäftsreisenden nach China, eingereicht von Linda Sanftenberg und Michaela Kramer, der Gruppe um Prof. Jörg Schelling, LMU München.

Burkhard Rieke, Düsseldorf, hat es wie jedes Jahr übernommen, die Vorträge der Jahrestagung zusammenzufassen (s. folgender Artikel). Wer an den Präsentationen, auch die der Preisträgerinnen, interessiert ist, möge sich bitte wegen des Zugangscodes bis 20. Oktober an die Geschäftsstelle wenden. Inhaltlich lag der Schwerpunkt eindeutig auf nicht infektiologischen Themen mit Bezug zur Versorgung und Betreuung vor, während und nach der Reise.

Erstmal hatten wir einer Jahrestagung einen Workshop für Studierende der Humanmedizin vorangestellt. Dieses Angebot ist von den Teilnehmenden als
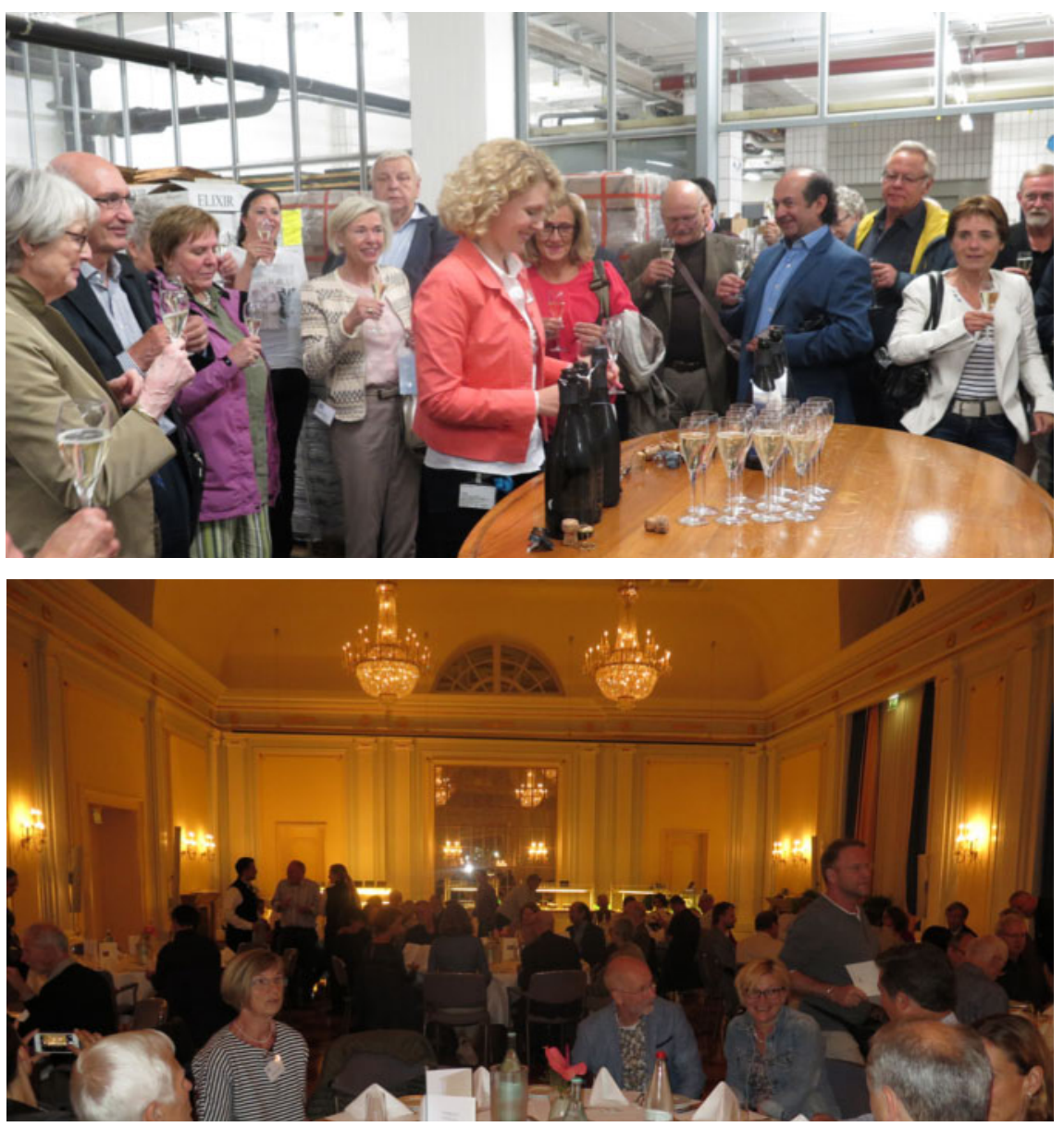

Gesellschaftsabend: Führung durch den Weinkeller und festliches Buffet im Gesellschaftshaus der Fa. BASF. 
,Einstieg، in die Reisemedizin mit großem Interesse angenommen worden. Herzlichen Dank an die Kollegen Ulrich Klinsing und Bernhard Wallacher, die diesen Workshop gestaltet und mit eigenen Kasuistiken bereichert haben. Wir werden diese Lehrveranstaltung künftig regelmäßig anbieten.

Die Abendveranstaltung war ein Highlight! Es ist den Programmverantwortlichen Rosemarie Mazzola und Bernhard Wallacher gelungen, eine Führung durch den beeindruckenden Weinkeller der Fa. BASF zu organisieren, dem sich ein festliches Buffet im Gesellschaftshaus dieses Unternehmens anschloss.

Die kommende Jahrestagung wird, abweichend von der bisherigen Praxis, voraussichtlich von Samstagmorgen, 23. September, bis Sonntagmittag, 24. September 2017, in Düsseldorf stattfinden. Damit soll den Kolleginnen und Kollegen, die davor den Kongress für Allgemeinmedizin in Düsseldorf besuchen wollen, Gelegenheit gegeben werden, beide Termine wahrzunehmen. Unsere nächste Jahrestagung wird ein besonderes Gepräge haben: 20 Jahre DFR und Vorstandswahl.

Die Tagung in Ludwigshafen markiert eine schmerzliche Zäsur in der Geschichte der DFR: Frau Ingrid Bergmann, Leiterin unserer Geschäftsstelle, wird im Laufe des kommenden Frühjahrs nach Erreichen des Rentenalters in den Ruhestand treten. Sie war somit zum letzten Mal für die Organisation unserer Jahrestagungen zuständig. Ingrid Bergmann war von Anfang an dabei und hat wesentlichen Anteil an der positiven Entwicklung zunächst des Fachverbands unter Prof. Kröger, dann der Fachgesellschaft in der jetzigen Form. In diesen nahezu 20 Jahren im Dienste der DFR war sie jederzeit eine kompetente und sehr verlässliche Ansprechpartnerin für alle Mitglieder und den jeweiligen Vorstand. Die DFR hat Ingrid Bergmann sehr viel zu verdanken; es war deshalb einstimmiger Beschluss des Vorstands und der anwesenden Mitglieder, Ingrid Bergmann die Ehrenmitgliedschaft anzutragen! Über die Nachfolge ist noch nicht entschieden. Ebenfalls mit besonderem Dank verabschiedet wurden die Tochter Christina und der Ehemann von Frau Bergmann; beide sind den Teilnehmern der Jahrestagungen seit vielen Jahren gut bekannt: bei der Anmeldung oder mit einem Fotoapparat bewaffnet.

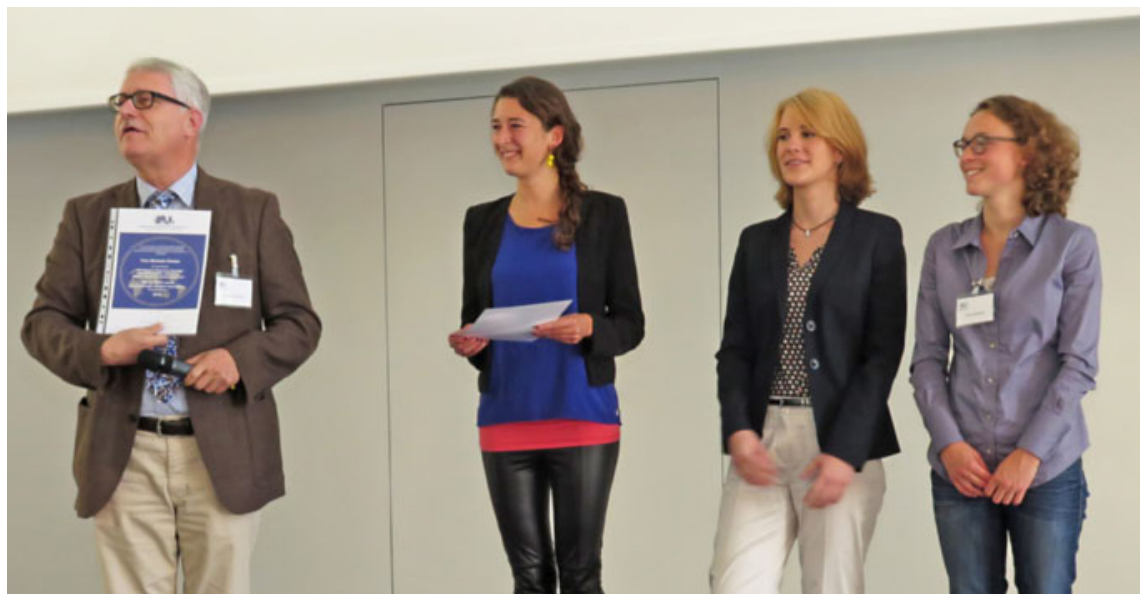

Prof. Günter Schmolz mit den drei Preisträgerinnen Linda Sanftenberg, Simone Kattner und Celina Drewell bei der Übergabe des Erich-Kroeger-und-Klaus-Jörg-Volkmer-Preises für Reisemedizin 2016.

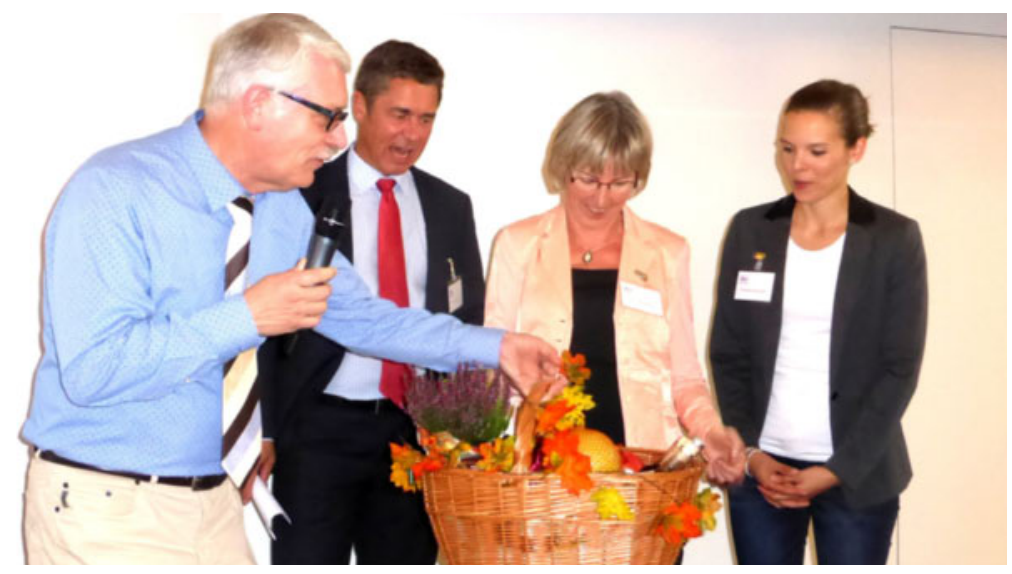

Prof. Günter Schmolz und Dr. Stefan Eßer verabschieden die langjährige Leiterin der Geschäftsstelle, Ingrid Bergmann, und ihre Tochter Christina.

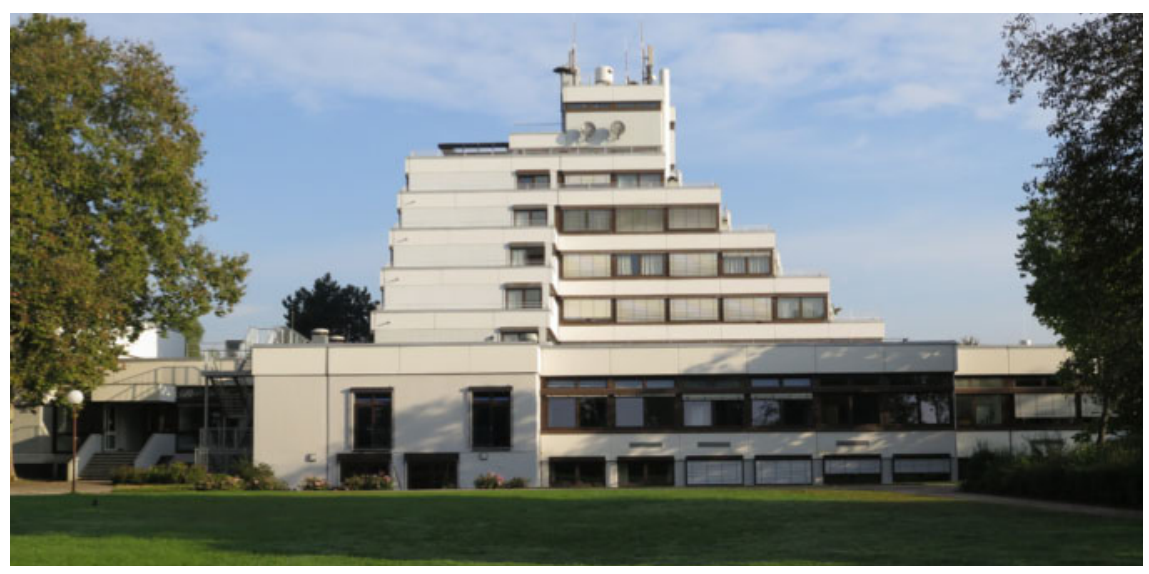

Tagungsort der 19. DFR-Tagung: Heinrich-Pesch-Haus in Ludwigshafen.

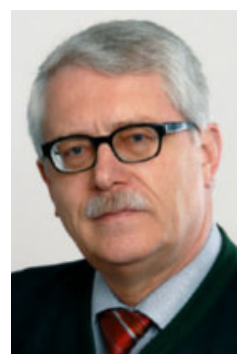

Mit den besten Grüßen Ihr 